\title{
Free Vibration Analysis of Electromechanical Integrated Electromagnetic Harmonic Movable Teeth Drive System
}

\author{
Yubo REN*, Weidong BIAN, Ruile YAN
}

\begin{abstract}
In the electromagnetic harmonic movable tooth drive system, the flexible wheel generates elastic deformation under the action of electromagnetic force, meshing output torque of the movable tooth and the center wheel. In view that the vibration of the flexible wheel under the action of electromagnetic force has an impact on the meshing of the movable tooth, which affects the output torque, this paper uses the theory of thin-shell vibration to simplify the flexible wheel into a thin shell with one end fixed at one end, and establishes an equilibrium equation of the flexible wheel displacement. And the vibration differential equation of the flexible wheel is derived. The influence of different parameters on its dynamic characteristics is analyzed. The theoretical values of several natural frequencies are compared with the ANSYS simulation values to verify the correctness of the method.
\end{abstract}

Keywords: electromagnetic harmonics; free vibration; flexible wheel; movable tooth

\section{AIMS AND BACKGROUND}

In the development and application of modern science and technology, on many occasions, low-speed output power or motion [1-3] is required, which is usually achieved by using a servo motor with a mechanical gear reducer [4]. The electromagnetic harmonic movable tooth drive proposed in this paper is a new type of transmission device that skillfully combines the mechanical movable tooth drive with the electromagnetic harmonics generated by the rotating electromagnetic field. The device can realize the low speed output of the large transmission ratio, and there are no high-speed rotating mechanical components in the device. Therefore, a faster response can be achieved with the characteristics of high precision, small volume, and light weight, etc., that has broad application prospects in the fields of electronic assembly, medical equipment, aviation and military [5].

In order to optimize the design parameters and effectively evaluate and control the dynamic performance of the system, it is necessary to study the electromechanical coupling dynamics theory of the transmission system.

\section{DIFFERENTIAL EQUATION OF FLEXIBLE VIBRATION}

The distribution of the internal force in the middle plane of the flexible wheel is shown in Fig.1.

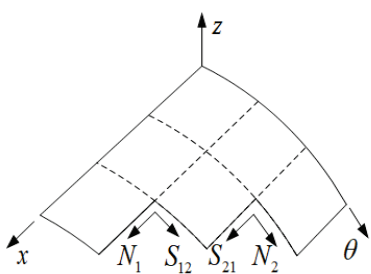

a)

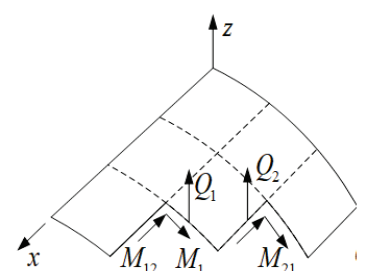

b)
Figure 1 Distribution of the internal force in the middle plane of the flexible wheel

In the $x$-section, the tension-compression internal force per unit length is $N_{1}$, the heavy force is $S_{12}$, the bending moment is $M_{1}$, the torque is $M_{12}$, and the transverse shear force is $Q_{1}$; in the $\theta$ section, the tension-compression internal force per unit length is $N_{2}$, the heavy force is $S_{21}$, the bending moment is $M_{2}$, the torque is $M_{21}$, the transverse shear force is $Q_{2}$. In which, Fig. 1a is the mid-plane internal forces of four forces, and Fig. $1 \mathrm{~b}$ is the curved internal forces of six forces.

At this time, the normal force of flexible wheels can be expressed as:

$$
F_{z}=\frac{\mu_{0} F_{1}^{2}}{4 \delta^{2}}[1+\cos 2(\omega t-\varphi)]
$$

wherein, $F_{1}$ is the amplitude of three-phase alternating current electromagnetic potential $\left(F_{1}=1.35 \times N k_{\mathrm{w}} I\right) . N$ is the number of turns per phase, $k_{\mathrm{w}}$ is the winding coefficient, $I$ is the current value; $\mu_{0}$ is the magnetic permeability $\left(\mu_{0}=4 \pi \times 10^{-7} \mathrm{H} / \mathrm{m}\right) ; \delta$ is the air gap length; $\omega$ is the current frequency; $t$ is the time; $\varphi$ is the current phase angle.

For a flexible wheel that is only subjected to radial loads, according to the Donne II thin shell theory [6, 7], the dynamic equation of the middle plane of the flexible wheel can be expressed as:

$$
\begin{aligned}
& \frac{\partial^{2} u}{\partial x^{2}}+\frac{(1-\mu)}{2} \frac{\partial^{2} u}{r^{2} \partial \theta^{2}}+\frac{(1+\mu)}{2} \frac{\partial^{2} v}{r \partial x \partial \theta}+\mu \frac{\partial^{2} w}{r \partial x}= \\
& =\frac{1}{K}\left(\rho h \frac{\partial^{2} u}{\partial t^{2}}-F_{x}\right) \frac{(1+\mu)}{2} \frac{\partial^{2} u}{r \partial x \partial \theta}+\frac{(1-\mu)}{2} \frac{\partial^{2} v}{\partial x^{2}}+\frac{\partial^{2} v}{r^{2} \partial \theta^{2}}+\frac{\partial w}{r^{2} \partial \theta}+ \\
& +k\left[\frac{(1-\mu)}{2} \frac{\partial^{2} v}{\partial x^{2}}+\frac{\partial^{2} v}{r^{2} \partial \theta^{2}}-\frac{\partial^{8} w}{\partial x^{2} \partial \theta}-\frac{\partial^{3} w}{r^{2} \partial \theta^{3}}\right]=\frac{1}{K}\left(\rho h \frac{\partial^{2} v}{\partial t^{2}}-F_{\theta}\right) \\
& \cdot \frac{12}{h^{2}}\left[\mu \frac{\partial u}{r \partial x}+\frac{\partial v}{r^{2} \partial \theta}+\frac{w}{r^{2}}-k\left(\frac{\partial^{3} v}{\partial x^{2} \partial \theta}+\frac{\partial^{3} v}{r^{2} \partial \theta^{3}}\right)\right]+\nabla^{2} \nabla^{2} w= \\
& =\frac{1}{D}\left(F_{z}-\rho h \frac{\partial^{2} w}{\partial t^{2}}\right)
\end{aligned}
$$

wherein, $u$ is the axial displacement, $v$ is the circumferential displacement, $w$ is the vertical mid-plane displacement, $h$ is the thin wall thickness of flexible wheel, $\mu$ is material Poisson's ratio, $r$ is the radius of middle plane; $F_{x}, F_{\theta}, F_{z}$ are the external forces acting on the flexible wheel in three directions. $\rho$ is the material density of flexible wheel, $k$ is the parameter; $k=\frac{h^{2}}{12 r^{2}}, K$ is the 
stiffness of material tensile, $K=\frac{E h}{1-\mu^{2}} ; D$ is the material bending stiffness $D=\frac{E h^{3}}{12\left(1-\mu^{2}\right)} ; E$ is the flexible wheel modulus, and $\nabla^{2}=\frac{\partial^{2}}{\partial x^{2}}+\frac{\partial^{2}}{r^{2} \partial \theta^{2}}$.

By using Donnell's hypothesis and ignoring external forces [8], Eq. (2) can be simplified as follows:

$$
\left.\begin{array}{l}
\frac{\partial^{2} u}{\partial x^{2}}+\frac{(1-\mu)}{2} \frac{\partial^{2} u}{r^{2} \partial \theta^{2}}+\frac{(1+\mu)}{2} \frac{\partial^{2} v}{r \partial x \partial \theta}+\mu \frac{\partial^{2} w}{r \partial x}=\frac{\rho h}{K} \frac{\partial^{2} u}{\partial t^{2}} \\
\frac{(1+\mu)}{2} \frac{\partial^{2} u}{r \partial x \partial \theta}+\frac{(1-\mu)}{2} \frac{\partial^{2} v}{\partial x^{2}}+\frac{\partial^{2} v}{r^{2} \partial \theta^{2}}+\frac{\partial w}{r^{2} \partial \theta}=\frac{\rho h}{K} \frac{\partial^{2} v}{\partial t^{2}} \\
\frac{12}{h^{2}}\left(\mu \frac{\partial u}{r \partial x}+\frac{\partial v}{r^{2} \partial \theta}+\frac{w}{r^{2}}\right)+\nabla^{2} \nabla^{2} w=-\frac{\rho h}{D} \frac{\partial^{2} w}{\partial t^{2}}
\end{array}\right\}
$$

\section{SOLUTIONS TO FREE VIBRATION}

Since the axial distribution of the cylindrical shell shape is close to the corresponding boundary condition beam mode function, the combination of the axial beam function and the circumferential trigonometric function can be used to approximate the mode shape function of the cylindrical shell. According to Rayleigh-Ritz beam function combination method $[9,10]$, the displacement of the flexible wheel in three directions can be assumed to be as follows:

$$
\begin{aligned}
& u=\sum_{m=1}^{P} A_{m} \frac{d X_{m}(x)}{d(x / l)} \cos n \theta \\
& \left.v=\sum_{m=1}^{P} B_{m} X_{m}(x) \sin n \theta \quad\left(\begin{array}{l}
n=0,1,2, \cdots \\
m=1,2,3, \cdots
\end{array}\right)\right\} \\
& w=\sum_{m=1}^{P} C_{m} X_{m}(x) \cos n \theta
\end{aligned}
$$

Wherein, the beam function is:

$$
X_{m}(x)=\cosh \lambda_{m} \frac{x}{r}-\cos \lambda_{m} \frac{x}{r}-x_{m}\left(\sinh \lambda_{m} \frac{x}{r}-\sin \lambda_{m} \frac{x}{r}\right)
$$

$A_{m}, B_{m}, C_{m}$ are the coefficients. These can be obtained according to the boundary conditions; $\mathrm{m}$ is the axial wave number, and the physical meaning indicates the wave number of the vibration mode in one circumferential length of the axial direction.

$\lambda_{m}=\frac{\varepsilon_{m} r}{l}, x_{m}=\frac{\cosh \varepsilon_{m}-\cos \varepsilon_{m}}{\sinh \varepsilon_{m}-\sin \varepsilon_{m}}$

The characteristic equation can be obtained by substituting Eq. (5) into Eq. (4).

$\Omega^{6}-K_{2} \Omega^{4}+K_{1} \Omega^{2}-K_{0}=0$
Wherein, $\Omega^{2}=\frac{\rho\left(1-\mu^{2}\right)}{E} \omega_{n}{ }^{2} r^{2}$ is frequency function.

$K_{2}=\frac{\lambda_{m}^{2}}{\delta_{m}}+\frac{1}{2}(3-\mu) n^{2}+1+\frac{1}{2}(1-\mu) \delta_{m} \lambda_{m}^{2}+$

$+k\left[n^{2}+2(1-\mu) \delta_{m} \lambda_{m}^{2}+\lambda_{m}^{4}-2 n^{2} \lambda_{m}^{2} \gamma_{m}+n^{4}+2 n^{2} \lambda_{m}^{2}(1-\mu)\left(\delta_{m}+\gamma_{m}\right)\right]$

$\delta_{m} K_{1}=\left[\lambda_{m}^{2}+\frac{1}{2}(1-\mu) \delta_{m} n^{2}\right]\left[n^{2}+\frac{1}{2}(1-\mu) \delta_{m} \lambda_{m}^{2}+1\right]+$

$+\frac{1}{2}(1-\mu) \delta_{m}^{2} \lambda_{m}^{2}-\mu^{2} \gamma_{m}^{2} \lambda_{m}^{2}-n^{2} \lambda_{m}^{2}\left[-\frac{\delta_{m}}{2}+\mu\left(\gamma_{m}+\frac{1}{2} \delta_{m}\right)\right]^{2}+$

$+k\left\{\left[\lambda_{m}^{2}+\frac{1}{2}(3-\mu) n^{2} \delta_{m}+\frac{1}{2}(1-\mu) \delta_{m}^{2} \lambda_{m}^{2}\right] \cdot\left[\lambda_{m}^{4}-2 n^{2} \delta_{m}^{2} \gamma_{m}^{2}+\right.\right.$

$\left.+n^{4}+2 n^{2} \lambda_{m}^{2}(1-\mu)\left(\delta_{m}+\gamma_{m}\right)\right]+\left[n^{2}+2(1-\mu) \delta_{m} n^{2}+\delta_{m}\right]$.

$\left.\cdot\left[\lambda_{m}^{2}+\frac{1}{2}(1-\mu) \delta_{m} n^{2}+\delta_{m}\right]-2 n^{2} \delta_{m}\left(n^{2}+\lambda_{m}^{2}\left[2(1-\mu) \delta_{m}-\gamma_{m} \mu\right]\right)\right\}$

$\delta_{m} K_{0}=\frac{1}{2}(1-\mu) \delta_{m} \lambda_{m}^{4}\left(1-\gamma_{m}^{2} \mu^{2}\right)+k\left\{\left[\frac{1}{2}(1-\mu) n^{2} \delta_{m}+\lambda_{m}^{2}\left(1-\gamma_{m}^{2} \mu^{2}\right)\right]\right.$

$\cdot\left[n^{2}+2(1-\mu) \delta_{m} \lambda_{m}^{2}\right]+\left[\left(\lambda_{m}^{2}+\frac{1}{2}(1-\mu) \delta_{m} n^{2}\right)\left(n^{2}+\frac{1}{2}(1-\mu) \delta_{m} \lambda_{m}^{2}\right)-\right.$

$-n^{2} \lambda_{m}^{2}\left[-\frac{\delta_{m}}{2}+\mu\left(\gamma_{m}+\frac{1}{2} \delta_{m}\right)\right]^{2}\left[\lambda_{m}^{4}-2 n^{2} \delta_{m}^{2} \gamma_{m}^{2}+n^{4}+2 n^{2} \lambda_{m}^{2}(1-\mu)\left(\delta_{m}+\gamma_{m}\right)\right]-$

$\left.\begin{array}{l}\left.n^{2}\left[\lambda_{m}^{2} \delta_{m} \gamma_{m}(1-\mu) \mu-2 \mu^{2} \lambda_{m}^{2} \gamma_{m}^{2}+2\left(\lambda_{m}^{2}+\frac{1}{2}(1-\mu) \delta_{m} n^{2}\right)\right] \cdot\right\} \\ \cdot\left[n^{2}+\lambda_{m}^{2}\left(2(1-\mu) \delta_{m}-\gamma_{m} \mu\right)\right]\end{array}\right\}$

Wherein,

$\delta_{m}=\frac{l}{\alpha_{m}^{2}} \int_{0}^{l}\left(X_{m}^{\prime}\right)^{2} \mathrm{~d} \alpha, \quad \gamma_{m}=\frac{l}{\alpha_{m}^{2}} \int_{0}^{l} X_{m}^{\prime \prime} X_{m} \mathrm{~d} \alpha$

\section{EXAMPLES AND DISCUSSION}

Based on the system parameters shown in Tab. 1, according to Eq. (6), the variation of natural frequency with flexible wheel parameters can be obtained by MATLAB, as shown in Fig. 2 to Fig. 5.

Table 1 System parameters
\begin{tabular}{|c|c|c|c|}
\hline Item & Value & Item & Value \\
\hline $\begin{array}{c}\text { Outer diameter of } \\
\text { stator(mm) }\end{array}$ & 130 & $\begin{array}{c}\text { Inner diameter of } \\
\text { stator(mm) }\end{array}$ & 80 \\
\hline Core length(mm) & 90 & Coil turns (turns) & 81 \\
\hline Polar logarithm (pair) & 3 & $\begin{array}{c}\text { Flexible wheel radius } \\
\text { (mm) }\end{array}$ & 39.5 \\
\hline $\begin{array}{c}\text { Effective length of } \\
\text { flexible wheel }\end{array}$ & 120 & $\begin{array}{c}\text { Thickness of flexible } \\
\text { wheel (mm) }\end{array}$ & 0.2 \\
\hline Air gap length & 0.5 & Working current I(A) & 3 \\
\hline
\end{tabular}

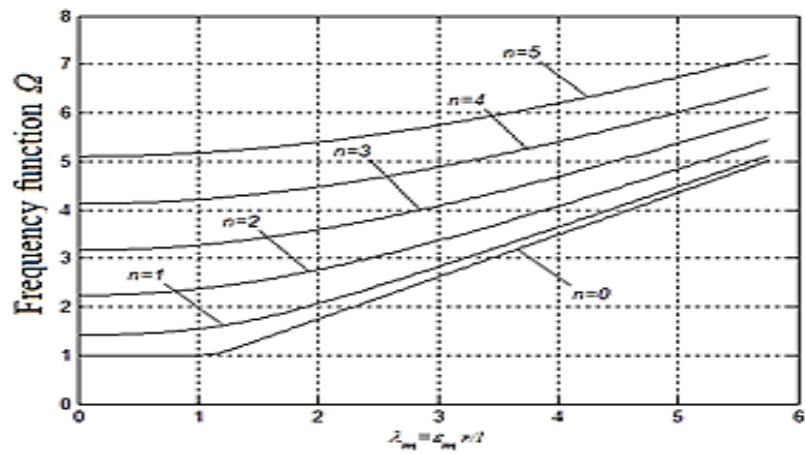

Figure 2 Frequency function curve under different circumferential wave numbers 


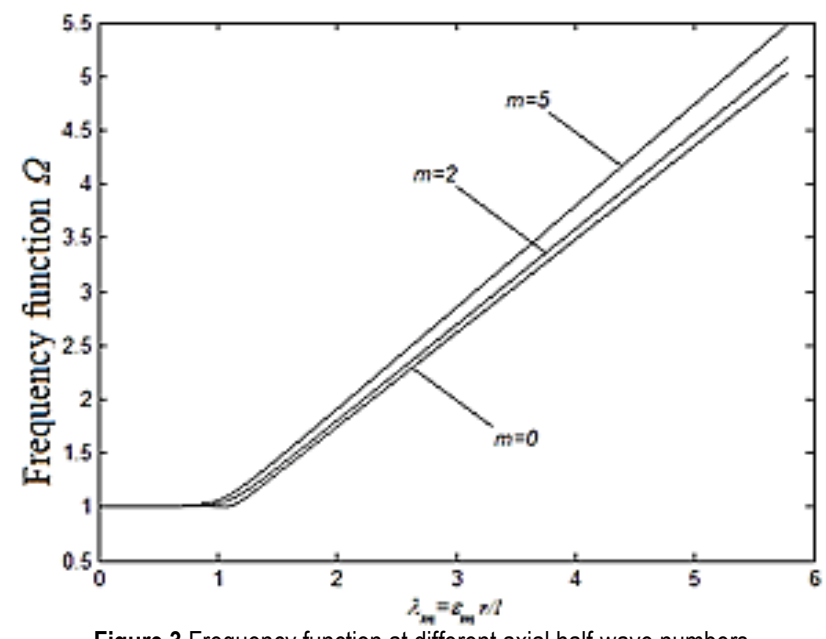

Figure 3 Frequency function at different axial half-wave numbers

(1) Fig. 2 is a frequency function curve under different circumferential wave numbers, from which it can be seen that the more circumferential wave numbers, the greater the value of frequency function, correspondingly, the higher the natural frequency.

(2) Fig. 3 also shows that the natural frequency increases with the increase of the axial half-wave number.

(3) The larger the ratio of wall thickness to radius of flexible wheel in Fig. 4, the higher the natural frequency of flexible wheel.

(4) The larger the ratio of radius to flexible wheel length in Fig. 5, the lower the natural frequency.

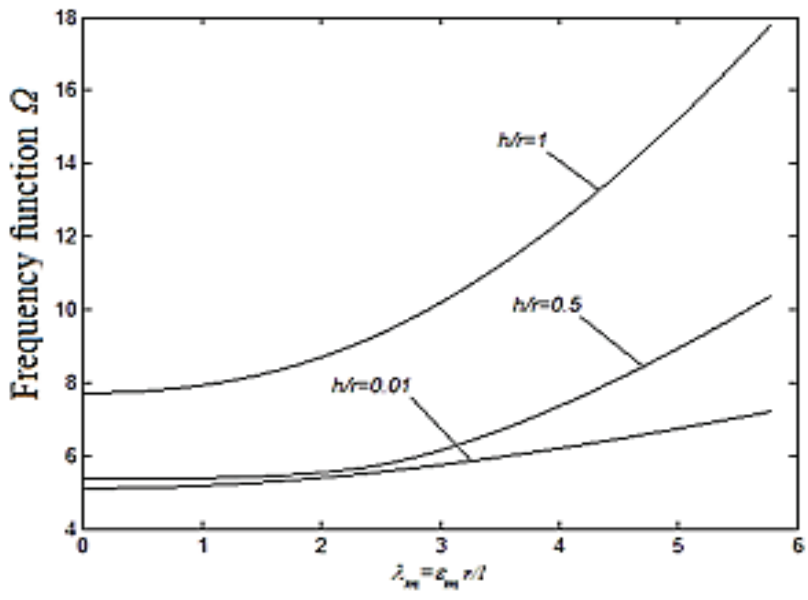

Figure 4 Influence curve of wall thickness on frequency function

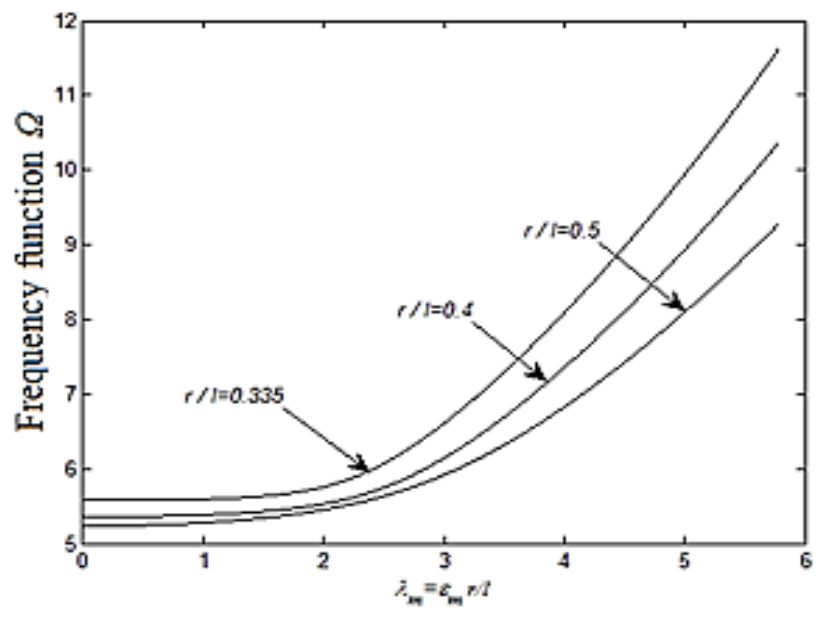

Figure 5 Influence curve of radius on frequency function
In addition, the natural frequencies of flexible wheel can be obtained from the above results. Since not all the values can be listed, two groups of flexible wheels are randomly selected for analysis in this paper. Tab. 2 shows the first five natural frequencies of flexible wheels when $m$ $=1, n=1$, and Tab. 3 shows the first five natural frequencies of flexible wheels when $m=4$ and $n=2$.

Table 2 The theoretical values of the first five natural frequencies of flexible wheels when $m=1$ and $n=1$

\begin{tabular}{|c|c|}
\hline Modal order & Natural frequency $/ \mathrm{Hz}$ \\
\hline 1 & 864.9 \\
\hline 2 & 886.3 \\
\hline 3 & 1046.8 \\
\hline 4 & 1077.2 \\
\hline 5 & 1553.6 \\
\hline
\end{tabular}

Table 3 The theoretical values of the first five natural frequencies of flexible wheels when $m=4$ and $n=2$

\begin{tabular}{|c|c|}
\hline Modal order & Natural frequency / Hz \\
\hline 1 & 946.6 \\
\hline 2 & 980.9 \\
\hline 3 & 1145.8 \\
\hline 4 & 1235.4 \\
\hline 5 & 1732.5 \\
\hline
\end{tabular}

As Tab. 2 and Tab. 3 indicate, the natural frequencies vary with the different axial and circumferential wave numbers.

Dynamic simulation of flexible wheel model is carried out by using finite element software of ANSYS. Eightnode shell element SHELL 93 is selected and divided into 15 elements along the bus direction of the thin shell and divide the circumference with this element size. Based on this element size, a total of 1440 elements and 4512 nodes can be obtained. Because of the symmetry of the structure, $1 / 4$ of the flexible wheels are selected to study. The obtained finite element model is shown in Fig. 6.

Define the error $X$ of theoretical value and simulation value as follows [11]:

$X=\frac{\text { theoretical value }- \text { ANSYS simulation value }}{A N S Y S \text { simulation value }} \times 100 \%$

When $m=1, n=1$ the theoretic value and simulation value of the first five natural frequencies are shown in Tab. 4.

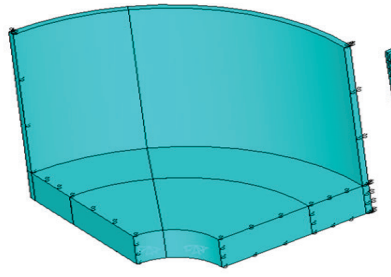

a)

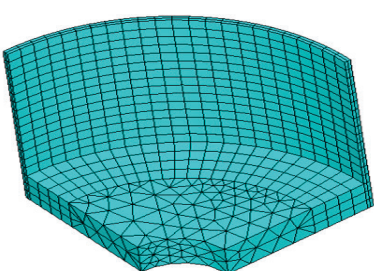

b)
Figure 6 Finite element model: a) network model, b) boundary conditions and forces

Table 4 The theoretic value and simulation value of the first five natural frequencies

\begin{tabular}{|c|c|c|c|}
\hline $\begin{array}{c}\text { Modal } \\
\text { order }\end{array}$ & $\begin{array}{c}\text { Theoretical value of } \\
\text { natural frequency / Hz }\end{array}$ & $\begin{array}{c}\text { Simulation value of } \\
\text { natural frequency / Hz }\end{array}$ & $X / \%$ \\
\hline 1 & 946.6 & 893 & 0.06 \\
\hline 2 & 980.9 & 899.9 & 0.09 \\
\hline 3 & 1145.8 & 946.7 & 0.21 \\
\hline 4 & 1235.4 & 980.4 & 0.26 \\
\hline 5 & 1732.5 & 1178.5 & 0.47 \\
\hline
\end{tabular}


It can be seen from the data in Tab. 4 that the theoretical value is slightly larger than the ANSYS simulation value, and the maximum error is only $0.47 \%$. Therefore, the numerical algorithm provided in this paper has certain accuracy.

\section{CONCLUSION}

The working principle of the electromagnetic harmonic movable teeth drives system is expounded. According to Donne II shell theory, the vibration differential equation of the system under the action of magnetic field is established. The frequency equation and the frequency solution of the flexible wheel with one end fixed and one end free are obtained. The natural frequency and the variation law of natural frequency with the flexible wheel parameters calculated by MATLAB, are also obtained. The obtained frequency values are in good agreement with the ANSYS simulation values.

By using the modal function in this paper, the free mode shape of flexible wheels can be obtained further, which lays a foundation for studying more dynamic characteristics of the system.

\section{Acknowledgments}

This paper is supported by National Natural Science Foundation of China. (No. 51875497).

\section{REFERENCES}

[1] Li, N., Wang, S. M., \& Du, J. J. (2013). Natural characteristics and dynamic load coefficient of power for embranchments gear transmission. Journal of Aerospace Power, 28(2), 445.

[2] Schier, M. \& Rinderknecht, F. (2013). Innovation examples for ecological vehicles based on aerospace research. Ecological Vehicles and Renewable Energies (EVER), 2013 $8^{\text {th }}$ International Conference and Exhibition on. IEEE, 1-7. https://doi.org/10.1109/EVER.2013.6521607

[3] Delucchi, M. A. \& Jacobson, M. Z. (2011). Providing all global energy with wind, water, and solar power, Part II: Reliability, system and transmission costs, and policies. Energy Policy, 39(3), 1170. https://doi.org/10.1016/j.enpol.2010.11.045

[4] Liu, F. H., Theodossiades, S., Bergman, L. A., Vakakis, A. F., \& McFarland, D. M. (2015). Analytical characterization of damping in gear teeth dynamics under hydrodynamic conditions. Mechanism and Machine Theory, 94(5), 141147. https://doi.org/10.1016/j.mechmachtheory.2015.08.007

[5] Ren, Y. B., Xu, L. Zh., \& Liang, Y. L. (2013). Analysis of flexible spline deformation in electromagnetic harmonic drive motor coupling systems. Journal of Yanshan University, 37(3), 211.

[6] Ren, Y. B., Xu, L. Z., \& Wu, Y. S. (2012). Study on Principle of Electromechanical Integrated Electromagnetic Harmonic Oscillating Teeth Transmission. International Journal of Advancements in Computing Technology, 4(16), 183. https://doi.org/10.4156/ijact.vol4.issue16.21

[7] Cao, Zh. Y. (1983). Theory of Plate and Shell Vibration. China Railway Publishing House, Beijing.

[8] Zh. L. X. U. (2006). Elasticity Mechanics, 2. $4^{\text {th }}$ Edition. Higher Education Press, Beijing.

[9] Q. Y, J. P. Fang. (1994). Analysis of Laminated Plates in Free Vibration by Beam Function. Journal of Wuhan Institute of Chemical Technology. 16(2), 7.
[10] Xu, L. Zh., \& Hao, X. H. (2012). Three DOF internal resonance for electromechanical integrated toroidal drive. Mechanism and Machine Theory, 58(3), 46. https://doi.org/10.1016/j.mechmachtheory.2012.07.007

[11] Park, S., Kim, S., \& Choi, J. H. (2018). Gear fault diagnosis using transmission error and ensemble empirical mode decomposition. Mechanical Systems and Signal Processing 108(4), 262. https://doi.org/10.1016/j.ymssp.2018.02.028

\author{
Contact information: \\ Yubo REN, Assoc. Prof., PhD \\ (Corresponding author) \\ School of Mechanical Engineering, \\ Yanshan University, \\ No 438 Hebei Street, Haigang District \\ Qinhuangdao City, Hebei Province, China 066004 \\ Weidong BIAN, Master's degree \\ School of Mechanical Engineering, \\ Yanshan University, \\ No 438 Hebei Street, Haigang District, \\ Qinhuangdao City, Hebei Province, China 066004 \\ Ruile YAN, Master's degree \\ School of Mechanical Engineering, \\ Yanshan University, \\ No 438 Hebei Street, Haigang District, \\ Qinhuangdao City, Hebei Province, China 066004
}

